\title{
Alte Traditionen und neue Tendenzen in der ungarischen Versicherungswirtschaft
}

\author{
von Eva Ebli*
}

\section{Einleitung}

Der Titel dieses Textes bieten einen breiten Spielraum für einen Gedankengang bezüglich "Schicksal" der Versicherung unter den sich mal schrittweise, mal stürmisch wandelnden wirtschaftlich-politischen Verhältnissen.

Obwohl heutzutage zweifelsohne diejenigen Reformprozesse im Mittelpunkt der Aufmerksamkeit im In- und Ausland stehen, die von den letztjährigen Regimewechseln der mittel- und osteuropäischen Ländern zutage gebracht worden sind, wäre es irreführend, das Thema nur auf dieses Geschehen begrenzt zu behandeln.

Die heutigen und zukünftigen Ereignisse haben - jedenfalls in Ungarn - ihre Wurzeln in den vergangenen Jahrzehnten; ohne die - sogar kurz - erwähnt zu haben, sind weder die Spannungen der jetzigen sozusagen Übergangsperiode, noch die gehofften zukünftigen Prozesse zu verstehen.

Deshalb habe ich einige - meines Erachtens nach geeignete - Aspekte gewählt, durch die sich die Einflußfaktoren der Versicherungsreform, das Verhältnis zwischen alten Traditionen und neuen Tendenzen, das Zusammenleben vom "nicht mehr" und "noch nicht" und die Spannungen der Übergangsperiode charakterisieren lassen.

Dabei kann man die in manchen Perioden der Wissenschaft zugeteilte Rolle - die herrschende Praxis vollkommen zu unterstützen - auch mitverfolgen.

Dies sind die folgenden:

- Rolle des Staates in der Wirtschaft allgemein und dessen Auswirkung auf die Versicherung.

- Die Versicherungsprodukte, als Widerspiegelung der wirtschaftlichen Rahmenbedingungen.

- Versicherer, als Anbieter vom Monopol zum Wettbewerb.

Dabei lassen sich die drei Aspekte voneinander kaum trennen; außerdem scheint, bevor wir in Details eingehen, ein allgemeiner Überblick, "eine Einführung", angebracht.

\footnotetext{
"Leiterin der Versicherungsaufsichtsbehörde, 1051 Budapest, Roosevelt-Platz 7-8.
} 


\section{Allgemeiner Überblick}

In der ungarischen Versicherungswirtschaft spielt sich ein Reformproze $\beta a b$, der zweifellos auch mit dem jetzigen wirtschaftlich-politischen Systemwechsel zusammenhängt. Es wäre aber irreführend, dessen Anfang zu einem bestimmten Zeitpunkt gebunden zu betrachten; es ist vielmehr so, daß man über Stufen sprechen soll, wobei von den einzelnen Faktoren des gesamten Versicherungswesens nicht immer das gleiche Tempo beibehalten worden ist. (So trat z. B. die Auflösung des Monopols definitiv am 1. 7. 1986 in Kraft; die Angebotspalette trug aber schon vorher und trägt zum Teil heute noch, Merkmale sowohl der alten Traditionen wie der neuen Tendenzen).

Die Versicherung als solche hat zweifelsohne alte Traditionen in Ungarn; doch soll diese Feststellung ergänzt werden, und zwar mit der etwas untergeordneten Rolle in den vergangenen Jahrzehnten, auf deren Grund im folgenden noch hingewiesen wird.

Der prozentuale Anteil des Prämienvolumens am Bruttoinlandprodukt, mit auch heute noch etwas unter $3 \%$, ist natürlich weit zurückgeblieben, nicht nur hinter den Ländern mit gut entwickelten Versicherungssystemen, sondern auch hinter den eigenen Möglichkeiten. Die Anzahl der Verträge ist - vor allem am Privatsektor - zwar hoch, doch oft bei niedrigen Versicherungssummen.

Die Branchenstruktur weicht immer noch vom westlichen Muster ab; bei einem unbefriedigenden Angebot der Lebens- und Krankenversicherung einerseits, sowie einem relativ großem Anteil der Versicherung agrarwirtschaftlicher Produkte andererseits, schien - bei Aufteilung des Gesamtgeschäftes in "Nicht-Leben" und "Leben" - das Schwergewicht bei dem ersterwähnten zu liegen. Dies hat sich in den letzten Jahren schrittweise geändert; im Jahre 1989 war das Verhältnis $71,5 \%$ zu 28,5\%.

Ansonsten ist die Angebotspalette - obwohl noch etwas schmaler - immer mehr dem westlichen Muster ähnlich.

Die Konsequenz des Aufwindes, sowie der stürmische wirtschaftlich-politische Wechsel der letzten Zeit, läßt sich für die Versicherung folgendermaßen summieren.

In Ungarn ist die Umwälzung stärker von dem Zwang der wirtschaftlichen Krise vorangetrieben worden. Dadurch ist die Notwendigkeit einer, die ganze Struktur umfassenden, Umwandlung in den Brennpunkt gestellt worden. Da einer der Schlüssel der Probleme im Bereich der Finanzen lag, sind schon anfangs der 80er Jahre übergreifende Reformen im Bank- und Versicherungswesen in Aussicht gestellt und schrittweise in die Tat umgesetzt worden.

Obwohl die Demonopolisierung im Jahre 1986 nur die Rolle haben sollte, die Erreichung der angestrebten Zicle (wie z.B. breitere Angebote, besserer Kundenservice, usw.) als Mittel zu fördern, stand sie in den vergangenen Jahren im Vordergrund der Aufmerksamkeit. (Nach vier Jahrzehnten Monopol läßt es sich viclleicht verstehen.)

Dazu kommt noch, daß3 man beinahe zur gleichen Zeit, als das Monopol aufgelöst worden ist, den Marktzutritt für Newcomer vom Ausland ermöglicht hat; in der Aufbauphase des Marktes jedoch nur in Form von Gründung oder Bctciligung an Gesellschaften mit Sitz in Ungarn. 


\section{Die Rolle des Staates in der Wirtschaft und deren Auswirkung auf die Versicherung}

Ungarn hatte lange Zeit eine Planwirtschaft, in der dem Staat während der ganzen Periode eine dominierende Rolle zugeteilt worden ist. Marktverhältnisse hatten einen stark eingeengten Raum; die Umverteilung der Einkommen wurde überwiegend auch als staatliche Funktion betrachtet.

Risikoempfindlichkeit und Risikobewußtsein überhaupt hatten eine sehr begrenzte Rolle, die sich natürlich auch auf die Bedeutung der Versicherung negativ auswirkte. Auch in der Wissenschaft waren die Themen Risiko und Risk-Management kaum vorzufinden.

Die breit ausgeschobenen Grenzen der staatlichen Aufgaben hatten eine mehrseitige Wirkung für die Versicherung. Um nur zwei Beispiele zu nennen:

- Versicherbarkeit der Risiken des staatlichen Eigentums,

- die fließenden Grenzen der staatlichen Subventionen im Zusammenhang mit den betriebswirtschaftlichen Forderungen gegenüber der Monopolanstalt, die sich im staatlichen Eigentum befand.

Beim staatlichen Eigentum gab es jahrzehntelang einen stark zentralisierten Entscheidungsprozeß, wobei praktisch alle Risiken vom Staat getragen waren. Dies hat das deklarierte Versicherungsverbot nach sich gezogen. Damit hat man praktisch auf eine beträchtliche Zeit die Industrie von der Versicherung ausgeschlossen, da das staatliche Eigentum in diesem Wirtschaftszweig das größte Gewicht gehabt hat. (Es ist übrigens trotz Förderung der Privatisierung auch heute noch der Fall.) Die Aufhebung des Versicherungsverbotes für das staatliche Eigentum fand im Jahre 1968 statt - im Zusammenhang mit der Wirtschaftsreform, in dessen Folge die staatlichen Unternehmen ihr Risiko schon auf eigene Kasse zu tragen hatten. Ab 1968 hat sich dann der erste fremde Gedanke - das eigene Risiko durch Versicherung zu decken - immer mehr bei staatlichen Firmen verbreitet und hat auch bei Klein- und Mittelbetrieben ab Anfang der 80er Jahre mehr und mehr zugenommen.

Unter den Theoretikern der Versicherungswissenschaft in den damaligen sozialistischen Ländern war eines der wichtigsten Diskussionsthemen die Versicherbarkeit des staatlichen Eigentums. Ursprünglich gab es eine allgemeine verneinende Meinung, die dann in mehreren Ländern - parallel mit der Stellungnahme der Praxis - revidiert worden ist.

Die staatliche Monopolanstalt funktionierte in unmittelbarem Verrechnungssystem mit dem Budget, wobei nicht der Gewinn, sondern das Wachstum der Beitragseinnahme die Hauptrolle gespielt hat. Dadurch war der Anreiz für die Sanierung verlustbringender Sparten nicht besonders stark, statt rein unternehmerischen Überlegungen waren sozusagen soziale und ähnliche Züge - mit Subventionscharakter - oft vorzufinden.

\section{Versicherungsprodukte als Widerspiegelung der wirtschaftlichen Rahmenbedingungen}

Hier möchte ich zwei Beispiele derjenigen Produkte erwähnen, die bei der Bevölkerung lange Zeit sehr verbreitet gewesen sind; durch ihren eigenartigen Aufbau läßt sich Harmonie und Spannung mit der wirtschaftlichen Umgebung ebenfalls beobachten. 
Es ist bekannt, daß es bis Ende der 60er, Anfang der 70er Jahre in Ungarn

- infolge der stark nivellierten Vermögens- und Einkommensverhältnisse eine relativ homogene Risikostruktur,

- stabile Preise,

- relativ breite Versorgung der Bevölkerung durch die staatliche Sozialpolitik gab.

Diese haben in der Versicherung eine spezielle betriebswirtschaftliche Abbildung mit sich gebracht: stark typisierte Formen der Deckung, Komplexität, vereinfachte Tarifierung.

Nach Anfangs-Harmonie gab es aber später immer mehr Spannungen bei Lösungen dieser Art, nicht zuletzt deshalb, weil dic - als Grundlage betrachteten - Bedingungen überholt waren.

Die ältesten Verträge der Gebäude- und Hausratversicherungen - die vergangenes Jahr noch die Mehrheit dieser Portefeuilles ausmachten - stammen vom Ende der 50er Jahre. Es waren

- Mehrrisikoversicherungen, wobei neben Risiken der Wohnung und des Hausrats auch ein Unfallrisiko gedeckt war,

- für unbestimmte Dauer,

- mit illimitierter Deckung und

- mit fixer Prämie, die sich nach der Zimmerzahl gerichtet hat.

Auch während der ursprünglich stabilen Verhältnisse waren manche Lösungen - z. B die für die Tarifierung gewählte Basis - diskutierbar. Doch sind mit Änderung der wirtschaftlichen Bedingungen (mit wachsender Streuung der Einkommen, steigender Inflation, usw.), auch in der Risikolage Änderungen eingetreten, so daß sich das Konzept kaum mehr halten ließ. Die ehemaligen Vorteile sind immer mehr zu Nachteilen geworden, vor allem beim Versicherer. Der Widerspruch zwischen unbestimmter Dauer und fixer Prämien einerseits und der wachsenden Inflation andererseits, machte diese Sparte von Jahr zu Jahr mehr verlustbringend. Man könnte aber auch den unlimitierten Deckungsumfang, oder die Zimmerzahl, als - mit der sich schrittweise entfaltenden Differenzierung der Vermögens- und Einkommensverhältnisse - immer mehr überholte Basis der Tarifierung erwähnen.

Obwohl die Aktualität des Wechsels schon früher bestand und die betriebswirtschaftlichen Methoden zur Auflösung dieser Spannungen bekannt sind, hat sich dieses prägnante Beispiel der alten Traditionen selbst überlebt. Die Versicherer sind mit dem neuen - den geänderten Verhältnissen angepaßten - Produkt erst im Herbst 1988 auf den Markt getreten.

Diese Verzögerung stand im Zusammenhang einerseits mit der Steigerung der Lebenshaltungskosten, infolge dessen mit der Verschlechterung des Lebcnsstandards zahlreicher Bevölkerungsschichten und demzufolge mit einer Aversion der Versicherer, diesen unpopulären Schritt durchzuführen. Andererseits lag die Priorität bei den Versicherern im wachsenden Marktanteil, gegenüber der Rentabilität der Produkte, umso mehr als in dieser Umstellungsphase die Solvabilitätsforderungen weder festgestellt noch berïcksichtigt wurden.

Das andere Beispiel ist ein "Schlagerprodukt" der 50/60er Jahre: Die sogenannte Gruppen-Lebens- und Unfallversicherung, die interessanterweise ähnliche betriebswirtschaftliche Elemente gehabt hat, wic das skizzierte Produkt im Bereich der Schaden- 
versicherung. Es handelt sich um eine kombinierte "Familienversicherung", die unter den aktiven Erwerbstätigen auf den Arbeitsplätzen vertrieben worden war. Die Prämie war auf Basis des Durchschnittsalters gerechnet und in einheitlichen Monatsbeträgen festgesetzt, die immer vom Lohn abgezogen wurde.

Um mit den damals noch langsam steigenden Lebenshaltungskosten Schritt halten zu können, erschien der Versicherer alle 6-8 Jahren mit einem "neuen Produkt", wobci eigentlich nur die Leistungen und die Prämien erhöht wurden. Das Produkt hatte einen starken Sozialversicherungs-Charakter; in den letzten Jahren sind die Nachteile immer eindeutiger geworden, vor allem die Gefahr der mangelnden Äquivalenz bei der Deckungsrückstellung. Aber auch davon abgesehen hat das erwähnte Vertriebssystem mit der wachsenden Anzahl der Klein- und Mittelbetriebe stark an Bedeutung verloren.

In ihrer jungen Zeit bedeutete diese Versicherung jedoch eine feste Konkurrenz für individuelle Lebensversicherungen, deren Entwicklung erst dann einen intensiven Schwung bekam, als der Vertrieb der erwähnten Typenversicherung mehr und mehr nachliess. In der letzten Zeit wird das Angebot an Lebensversicherungen - vor allem an Rentenversicherungen - immer vielfarbiger, doch ist deren Vertrieb - trotz Förderung der Ersparnisse - wegen der hohen Inflationsrate keine leichte Aufgabe.

\section{Versicherer: als Anbieter vom Monopol zum Wettbewerb}

Wie schon erwähnt, wurde das Versicherungsmonopol in Ungarn am 1. 7.1986 "de jure" aufgelöst und damit das Feld für Wettbewerb geschaffen, doch "de facto" läßt die Entfaltung der Marktmechanismen noch auf sich warten. Es wäre im Moment noch irreführend, über einen Markt zu sprechen, eher über Anfänge eines sich formulierenden Marktes, wo Konturen des Wettbewerbes schon zu finden sind.

Als "rechtlicher Nachfolger" der einstigen staatlichen Monopolanstalt sind zum gleichen Zeitpunkt - durch Teilung des Portefeuilles - 2 staatliche Gesellschaften gegründet worden. Die Privatisierung als wichtiger weiterer Aspekt der Versicherungsreform kam erst etwas später dazu - als Teil der allgemeinen Tendenz zur Reduzierung des staatlichen Eigentums.

\section{Newcomer am ungarischen Versicherungsmarkt}

Die Öffnung des ungarischen Marktes hat selbstverständlich auch das Interesse ausländischer Versicherer sowie Makler geweckt. Es ist vielleicht interessant, einiges über Versicherer und über Makler zu sagen.

Versicherungstätigkeit kann in Ungarn in Form von Aktiengesellschaften, von Versicherungsgenossenschaften und von Versicherungsvereinen auf Gegenseitigkeitsbasis - in all diesen Fällen nur mit Sitz in Ungarn - betrieben werden.

Die Gründung sowie jegliche Form der Einbeziehung ausländischen Kapitals wird durch die Regierung zugelassen.

Heute sind auf dem ungarischen Markt sieben Aktiengesellschaften und acht Versicherungsvereine auf Gegenseitigkeitsbasis tätig; zwei davon sind die staatlichen Unternehmen, die Anfang 1990 in AG's umgewandelt wurden. 
Als Neugründung zählen fünf Gesellschaften, von denen in vier ausländisches Kapital - namentlich deutsches, amerikanisches, österreichisches, italienisches - repräsentiert ist.

Die Präsenz des ausländischen Kapitals ist zum Teil bei der Gründung, zum Teil in Form von Beteiligung durch Ankauf von Aktien zustande gekommen. In der Mehrheit der Joint Ventures ist der ungarische Partner auch Versicherer, wie z. B. im Falle von ABGenerali (AB-Erste Allgemeine-Generali). Gegenbeispiele sind:

- "Providentia", wobei die Gründer die "Wiener Allianz", die "Ungarische Post" sowie die "Postsparkasse" sind;

- die Erste Amerikanisch-Ungarische Versicherungs AG, die auf Partnerschaft der A. I. U., der ungarischen Genossenschaftsbank und mehreren Gewerkschaften aufgebaut ist.

Diese Gesellschaften sind überwiegend sogenannte Kompositversicherer, obwohl nicht alle Sparten von allen betätigt werden. Zu dieser Zeit ist die Spartentrennung nicht deklariert.

Der Markt hat nach wie vor einen stark oligopolistischen Charakter. Die zwei großen, einst staatlichen Gesellschaften - die $98 \%$ der Beitragseinnahmen ausmachen - sind an 3 weiteren Gesellschaften beteiligt.

Ich glaube, es soll nicht besonders dafür argumentiert werden, daß dem Ausbau des Marktes, vor allem durch die wachsende Anzahl der unabhängigen Marktteilnehmer also Neugründung in absolutem Sinne - geholfen wäre. Das Risiko der jungen Gesellschaft ist in diesem Falle unbestritten am größten, besonders wenn man an die geringe Anzahl Fachkräfte und an das eventuell fehlende Vertriebsnetz denkt.

Die Versicherungsvereine auf Gegenseitigkeitsbasis sind keine Neugründungen. Diese Form scheint weiterhin innerhalb des betriebsinternen Rahmens zu bleiben, wobei die Versicherten mit den Mitgliedern identisch sind. Es ist jedoch nicht auszuschließen, daß sich im Laufe der Reform der Sozialversicherung Versicherungsvereine als quasi 2. Säule des Systems entfalten werden.

In den letzten Monaten haben, sowohl auf einheimische wie auch auf ausländische Initiative, unabhängige Maklerfirmen ihre Tätigkeit begonnen. Diese Form von Vertrieb ist damit seit Jahrzehnten wieder auf dem Markt erschienen. Eine Maklertätigkeit kann nur in einer Gesellschaft mit Rechtspersönlichkeit ausgeführt werden.

An der Eigenart des ungarischen Marktes - nämlich, daß es in der Monopolzeit und auch beim Anfangstadium des Wettbewerbs keine Makler im üblichen Sinne gab - darf man nicht vorbeigehen. Die - beim Massengeschäft überwiegend einfachen, stark typisierten - Verträge wurden zum größten Teil durch das Vertriebsnetz verschiedener Unternehmen, Sparkassen, Spargenossenschaften, Reisebüros usw. abgeschlossen, d. h. auf Provisionsbasis mit einer gegenseitigen Vereinbarung. In den meisten Fällen waren solche Aufträge nur für einzelne Funktionen gegeben, manchmal waren aber mehrere - Vertrieb, Inkasso, Regulierung von kleineren Schäden - mitinbegriffen.

Insgesamt kann man sagen, dass es die einfach aufgebauten Verträge ermöglicht haben, eine oder mehrere Aufgaben auf Außenstehende, nebenberuflich Tätige zu übertragen. In den meisten Fällen war es irgendwie im Zusammenhang mit der Grundtätigkeit, z. B. Kreditgewährung für Haushalt- und Hausratversichcrung bei der Sparkasse. 
Dies hat auch Vor- und Nachteile gehabt. Es hat durch die erhöhte externe Kapazität eine enorme Entwicklung ermöglicht, mit einer günstigen Kostenquote. Gleichzeitig sind aber Kundenpflege und Kundenservice stark im Hintergrund geblieben; Versicherung wurde etwas unpersönliches. Mit dem sich langsam entfaltenden Wettbewerb wurde es immer mehr zu einem Engpaß.

Als eine überraschende Erscheinung ist zu begrüßen, daß die Zahl der Makler - wie erwähnt - in den letzten Monaten schnell gewachsen ist. Es gibt Beispiele für rein ungarische, wie auch für rein ausländische Gründungen oder sogar Joint Ventures; dabei handelt es sich überwiegend um GmbH's. 